\title{
A família Agaricaceae Chevall. em trechos de Mata Atlântica da Reserva Biológica do Tinguá, Nova Iguaçu, Rio de Janeiro, Brasil: Gêneros Agaricus, Cystolepiota e Lepiota ${ }^{1}$
}

\author{
Margéli Pereira de Albuquerque $2,4,5$, Antônio Batista Pereira ${ }^{3}$ e Aníbal Alves de Carvalho Júnior ${ }^{4}$
}

Recebido em 7/05/2009. Aceito em 29/03/2010

\begin{abstract}
RESUMO - (A família Agaricaceae Chevall. em trechos de Mata Atlântica da Reserva Biológica do Tinguá, Nova Iguaçu, Rio de Janeiro, Brasil: Gêneros Agaricus, Cystolepiota e Lepiota). Agaricaceae é a família tipo da ordem Agaricales e apresenta espécies de reconhecida importância nutricional e econômica. O principal objetivo deste trabalho foi o levantamento das espécies de Agaricaceae em trechos de Mata Atlântica da Reserva Biológica do Tinguá, nas delimitações do Município de Nova Iguaçu, Rio de Janeiro. Serão apresentadas as espécies dos gêneros Agaricus, Cystolepiota e Lepiota coletados nas diferentes formações vegetais na Reserva Biológica do Tinguá. Foram registrados os seguintes táxons: Agaricus subrufescens Peck., A. dulcidulus Schulzer., Lepiota lilacea Bresadola., L. tepeitensis Murril., L. forquignoni Quélet., L. echinela var rhodorhiza (Romagn. \& Locq. ex. Orton) Hardtke \& Rödel., L. subincarnata Lange e Cystolepiota seminuda (Lasch) Bon. São apresentadas chaves dicotômicas para identificação de espécies; descrições, fotos de hábito, imagens de microscopia, ilustrações, distribuição, habitat, dados relativos às épocas de surgimento dos basidiomas e importância das espécies quando conhecida.
\end{abstract}

Palavras-chaves: Agaricales, Micobiota, Mata Atlântica, Rio de Janeiro

\begin{abstract}
The family Agaricaceae Chevall. in the Tinguá Biological Reserve rain forest, Nova Iguaçú, Rio de Janeiro, Brazil: Genera Agaricus, Cystolepiota and Lepiota). Agaricaceae is the type family of Agaricales and its species are of nutritional and economic importance. The main aim of this survey was to describe the Agaricaceae species of rain forest patches in the Tinguá Biological Reserve, located in the vicinity of Nova Iguaçu, Rio de Janeiro state, Brazil. Species of the genera Agaricus, Cystolepiota and Lepiota, collected in different plant formations, are presented. The following taxa were recorded: Agaricus subrufescens Peck., A. dulcidulus Schulzer., Cystolepiota seminuda (Lasch) Bon, Lepiota. lilacea Bresadola., L. tepeitensis Murril., L. forquignoni Quélet., L. echinela var rhodorhiza (Romagn. \& Locq. ex. Orton) Hardtke \& Rödel and L. subincarnata Lange. A species key, descriptions, habit photo, microscopic features, distribution and species importance (when known) are given.
\end{abstract}

Key words: Agaricales, Mycobiota, Atlantic rain forest, Rio de Janeiro.

\section{Introdução}

A familia Agaricaceae é de grande interesse científico, possui espécies envolvidas em processos biotecnológicos, espécies de importância gastronômica e outras com propriedades medicinais (Silva \& Esposito, 2004; Eira 2004; Didukh et al. 2003). Segundo Kirk et al. (2001), a família compreende 51 gêneros e 918 espécies.incluindo gêneros gasteróides e secotiódes. Apesar da importância ecológica e econômica da família poucos inventários de Agaricaceae foram realizados no estado do Rio de Janeiro, dentre estes pode ser citado as coletas realizadas por Jezek (1973) no município de Cabo Frio e as coletas realizadas por Ule e identificadas por Paul Hennings, que encontram-se depositadas no herbário da Universidade de Hamburgo na Alemanha (Friederichesen 1973). Este trabalho apresenta os primeiros resultados em relação a diversidade da família Agaricaceae na Reserva Biológica do Tinguá.

\section{Material e métodos}

A Reserva Biológica do Tinguá (REBIO-Tinguá) localiza-se no estado do Rio de Janeiro, a área da Reserva abrange os Municípios de Nova Iguaçú, Miguel Pereira, Petrópolis e Duque de Caxias (Saracura,1996). A Unidade de Conservação está no limite norte da Baixada Fluminense, distante cerca de $70 \mathrm{~km}$ da cidade do Rio de Janeiro. Segundo Golfari \&
Moosmayer (1980), a partir do século XVI as Florestas da Baixada Fluminense, começaram a ceder dando lugar ao cultivo de cana-de-açúcar. Porém, a área que atualmente compreende a Rebio Tinguá possui remanescentes de Mata Atlântica em excelente estado de conservação. Segundo o Relatório técnico-científico do Instituto de Pesquisas Jardim Botânico do Rio de Janeiro (IPJBRJ 2002), a conservação desta região deve-se a um dos primeiros atos de preservação da natureza, quando D. Pedro II, declarou como Floresta Protetora uma porção de mata que abrangia a atual área da Rebio Tinguá. A finalidade era de proteger importantes mananciais para o abastecimento da cidade do Rio de Janeiro. Mais recentemente essa porção de mata foi transformada em unidade de conservação pelo decreto 97.780 , de 23 de maio de 1989, denominada então Reserva Biológica do Tinguá. A diversidade florística nas Florestas Montanas da REBIO é relativamente alta quando comparada com outros trechos de Mata Atlântica. A riqueza registrada é superior quando comparada a outros trechos de floresta bem estudadas nos trópicos como Macaé de Cima, no estado do Rio de Janeiro (Guedes-Bruni et al. 1997) e Reserva Duque, no estado do Amazonas (Ribeiro et al. 1999).A reserva possui um alto grau de endemismos, com elevado número de espécies exclusivas das florestas da porção central da Serra do Mar, ou mesmo exclusivas do Maciço do Tinguá (IPJBRJ 2002).

As altitudes variam de próximas ao nível do mar até cerca de $1600 \mathrm{~m}$ (Pico do Tinguá).Os solos são dos tipos cambissolos, latossolos e podzólicos freqüentemente associados entre si e a solos litólicos com afloramentos de rochas. Os tipos dominantes são os podzólicos oriundos de rochas granítico-gnaissicas do pré-cambriano (RADAM Brasil 1983).O clima é quente úmido com estação seca pouco definida nos meses de julho e agosto, correspondendo ao tipo de Am (Koeppen 1948). A temperatura média anual é de $21,6^{\circ} \mathrm{C}$ e a máxima absoluta é de $40^{\circ} \mathrm{C}$, geralmente ocorrendo no mês de janeiro. A precipitação anual é de 2099,3mm e os meses de dezembro e janeiro são os mais chuvosos (Koeppen 1948).

\footnotetext{
1 Parte da dissertação de Mestrado do primeiro Autor

2 Universidade Federal de Pelotas, Instituto de Biologia, Departamento de Microbiologia e Parasitologia, Pelotas, RS, Brasil

3 Universidade Federal do Pampa, Campus São Gabriel, RS, Brasil

4 Instituto de Pesquisas jardim Botânico do Rio de Janeiro, Rio de Janeiro, RJ, Brasil

5 Autor para correspondência: margeli_albuquerque@hotmail.com
} 
O material estudado foi coletado durante 4 (quatro) excursões de campo com duração de 3 a 4 dias cada, no período de dezembro de 2003 a dezembro de 2004. As coletas seguiram os métodos para estudos de Agaricales com atenção especial aos caracteres morfológicos dos basidiomas frescos (Fidalgo \& Bononi 1984; Pereira \& Putzke 1990). O método de amostragem utilizado foi o de coletas em áreas de borda e interior de mata, em ambientes favoráveis ao desenvolvimento de basidiomas, nos principais trechos da área de estudo, compreendendo as formações vegetais de Floresta Aluvial, Submontana e Montana (Veloso et al. 1991).

Em campo foram realizados registros fotográficos do hábito, registros de detalhes do basidioma e ilustrações. As coordenadas geográficas foram obtidas com GPS (Garmim 1200). Os materiais foram coletados com cuidado para não danificar estruturas importantes para identificação das espécies. Substratos como folhas, madeiras ou musgos foram coletados juntamente com os basidiomas para manter a umidade das amostras e compor as exsicatas. Os espécimes foram acondicionados individualmente em sacos de papel de 25 x $10 \mathrm{~cm}$ e levados ao laboratório.
Para o estudo das estruturas microscópicas foram confeccionadas lâminas semi-permanentes. Os cortes para observação do himênio, trama da lamela e camada cortical foram realizados com lâmina de barbear, a mão livre sob microscópio estereoscópico. O Tratamento com Vermelho Congo foi utilizado para corar as paredes das hifas e a reação de Schaeffer \& Müller para identificar seções do gênero Agaricus (indicada no texto pelos símbolo RS+, quando positiva ou RS-,quando negativa). Os caracteres observados foram ilustrados e fotografados. As imagens de microestruturas foram obtidas com auxílio de câmera microscópica Motic 2000 acoplada a Microscópio óptico Zeiss e Câmera CoolSnap (Sony) acoplada ao microscópio óptico Olympus. As mensurações foram realizadas com os programas Image Pró plus e Motic Image plus. As coletas foram depositadas no Herbário do Instituto de Pesquisas Jardim Botânico do Rio de Janeiro (RB).

\section{Resultados e discussão}

Chave para espécies dos gênero Agaricus, Cystolepiota e Lepiota encontradas na Rebio Tinguá

1. Basidiósporos pigmentados

2. Píleo fibriloso-esquamuloso, coloração vinácea 1. A. dulcidulus

2. Píleo branco, com escamas marrom douradas .2. A. subrufescens

1. Basidiósporos 3

3. Camada cortical do píleo formada por um epitélio constituído por exclusivamente por esferocistos 3. C. seminuda

3. Camada cortical do píleo não epitelial, mas eventualmente com alguns esferocistos (principalmente no ápice dos espinhos), ou com hifas prostadas e em alguns pontos eretas formando escamas fibrilosas ou algodonosas; em algumas espécies formando himenioderme no disco central, mas mais frequentemente tricodermial

4. Basidiósporos elípticos 6. L. lilacea

4. Basidiósporos ovais 5

5. Basidioma médio a grande, diâmetro do píleo maior que $6 \mathrm{~cm}$

8. L. tepeitensis

5. Basidioma pequeno, geralmente com diâmetro do píleo menor que $5 \mathrm{~cm}$ .66

6. Camada cortical formada por hifas eretas, finas, como uma paliçada

5. L. forquignonii

6. Camada cortical do píleo formada por hifas tricodermiais e himenodermais . .7

7. Elementos da camada cortical subcilíndricos; anel ausente

7. L. subincanata

7. Presença de elementos subglobosos a esferopedunculados na camada cortical do píleo; anel presente 4. L. echinella var rhodorhiza

1. Agaricus dulcidulus Schulzer, Icon. Sel. Hymenomyc. Hung.: 29. 1874.

(Fig. 1)

Píleo 3,7-4,5 cm, vináceo, cônico no inicio tornando-se campanulado a aplanado, superfície fibrilosa-escamosa, borda involuta. Lamelas livres, colariadas, brancas a creme-claras, tornando-se marrons após desidratação, próximas entre si. Estipe 3-8,3 x 0,2-0,5 cm, cilíndrico, reto, fistuloso, branco, não mudando de cor ao toque, base com bulbo basal marginado. Anel presente, branco, ascendente, posicionado no terço superior. Hábito gregário. Basidiósporos 4-5 x 2,7-4 $\mu \mathrm{m}$, hialinos a marrons, ovais, parede dupla, poro germinativo ausente; hilo excêntrico, inamilóide em reagente de Melzer. Basídios 13-16,7 x 5-6,9 $\mu \mathrm{m}$, clavado-ventricoso, hialinos, com 4 esterigmas. Pleurocistídios ausentes. Queilocistídios 35-37 x 12-18 $\mu \mathrm{m}$, claviformes. Camada cortical formada por hifas cilíndricas, eretas, septadas, hialinas a marrom-avermelhadas, formando uma tricoderme. Fíbulas ausentes.

Habitat: Crescendo em ninho de formigas no interior da mata.

Material examinado: Brasil. Rio de Janeiro Nova Iguaçú, REBIO-Tinguá, Estrada do Colomi. 22 $35^{\circ}$ 03.4'S$43^{\circ} 24^{\prime} 47.4^{\prime \prime} \mathrm{W}$, alt $97 \mathrm{~m}, 14 / \mathrm{X} / 2004$, Albuquerque 36-III, (RB417262).

Distribuição: Ásia: Rússia, Azerbaijão, Japão, Israel (Wasser 2002) América do Norte: Canadá, EUA. América do Sul: Argentina, Bolívia, Brasil e Venezuela (Heinemann 1962c) e no presente trabalho. Caribe: Trinidad, Tobago (Pegler 1983).Europa: Reino Unido, Dinamarca, França, Itália, Alemanha, Rússia, Ucrânia, Polônia, República Tcheca, Hungria (Cappelli 1984).

Comentários: O ambiente onde esta espécie foi coletada lembra o habitat de Leucoagaricus gongylophorus (Möelller) 
Heim. por crescer em formigueiro e possuir hábito gregário (Fisher et al. 1994). Singer (1986) cita que espécies do gênero Agaricus também podem apresentar hábito termitófilo. Os basidiósporos marrons permitiram diferenciar esta das espécies do gênero Leucoagaricus principalmente porque os basidiósporos de Leucoagaricus são sempre claros, às vezes um pouco pigmentados, (em L gongylophorus são hialinos) mas nunca marrons e com parede grossa como em Agaricus. O espécime analisado caracteriza-se por possuir basidiomas delgados, vináceos, Basidiósporos ovóides e queilocistídios piriformes. Enquadra-se na descrição de A. purpurellus (F.H. Møller) F.H. Møller apresentada por Pegler (1983) e Cappelli (1984) e concorda com o material homônimo identificado por Pegler (1997). Nauta (2001) considera $A$. purpurellus como uma variante menor de $A$. dulcidulus, não apresentando outros caracteres que justifique a separação entre os taxa, por esta razão A. purpurellus é considerado como sinônimo de $A$. dulcidulus, sendo este último o nome válido para esta espécie (Kirk et al. 2001). A. dulcidulus, pode ser confundido com $A$. singeri Heinem., mas nesta espécie os queilocistídios são ausentes.

Importância: Segundo Cappelli (1984), a espécie é comestível.

\section{Agaricus subrufescens Peck, New York State Mus. Ann. Rep. 46: 105. 1893.}

(Fig. 2)

Píleo 7,5 cm, carnoso, campanulado, umbo plano, escamas concêntricas marrom-douradas densamente arranjadas sobre a região central do píleo, contexto branco, margem involuta. Lamelas livres, rosadas, lanceoladas, arqueadas. Estipe $11 \mathrm{x}$ $2 \mathrm{~cm}$, cilíndrico, sólido, branco, superfície esquamulosa, fibroso, imutável ao toque, base bulbosa com rizóides. Anel no terço superior, ascendente depois pendente, branco, simples, membranoso, com escamas marrons. Hábito solitário. Basidiósporos 5,4-7,8 x 3,4-4,2 $\mu \mathrm{m}$, méleos, elípticos, hilo excêntrico, inamilóides em reagente de Melzer. Basídios 14-16 x $8 \mu \mathrm{m}$, hialinos, clavados, com 2-4 esterigmas. Trama himenoforal irregular. Pleurocistídios ausentes. Queilocistídios 16,8-20 x 5,9-7,8 $\mu \mathrm{m}$, clavados com apêndice lageniforme, claviformes com apêndice sub-captado. Camada cortical formada por hifas cilíndricas, prostradas, septadas, às vezes apresentando elementos globosos no ápice das hifas. RS-. Fíbulas ausentes.

Hábitat: Crescendo no solo, na borda da mata

Material examinado: Brasil. Rio de Janeiro: Nova Iguaçú, REBIO-Tinguá, Estrada do Colomi. 22³4'59.6”S4326'45.8”'W, alt. 597m, 16-X-2004, Carvalho Jr. et al. 55-III (RB-417276).

Distribuição geográfica: América do Sul: Brasil (Heinemann 1993; Rick 1930; Wasser et al. 2002) e no presente trabalho. América do Norte: EUA, Hawai. Europa: Ucrânia (Wasser 2002).

Comentário: A presente amostra se enquadra na descrição de $A$. brasiliensis presente em Wasser et al (2002). Wasser et al. (2002) propõem o nome $A$. brasiliensis como uma espécie nova baseada em A. blazei Murril determinado por Heinemann (1993) e em amostras coletadas no estado do Paraná. Desde então esta espécie não havia sido recoletada. Kerrigan (2005) concorda com Wasser et al. (2002) que o material proveniente do Brasil é diferente de A. blazei Murril, mas não a confirma como espécies nova. Este autor realizou um estudo comparativo utilizando caracteres morfológicos e moleculares de espécimes herborizados e cultivados em laboratório de amostras de A. blazei, A. brasiliensis, $A$. subrufescens e $A$. rufotegulis Nauta, e considerou-as um grande complexo de espécies. Neste contexto A. blazei é tratada como entidade taxonômica independente das outras três espécies, que foram sugeridas como pertecentes a um único táxon. Nesse caso seguindo as normas de nomenclatura botânica o nome correto para $A$. brasiliensis e $A$. rufotegulis é $A$. subrefescens, uma vez que este é o nome mais antigo, sendo os nomes anteriores seus sinônimos. $A$. subrufescens Peck foi citada primeiramente por Rick (1930) como Psaliotta subrufescens Peck, para o município de São Leopoldo, Rio Grande do Sul, porém com basidiósporos menores ao da descrição original. Rick (1939) sugere uma variedade de $A$. subrufescens como "varietas microspora", considerada por Kerrigan (2005) como uma designação sem valor taxonômico e que, desta forma, os espécimes de Rick não foram validamente publicados. As descrições publicadas por Rick $(1930 ; 1939)$ não são suficientes para efeitos de comparação com o material coletado na RebioTinguá, e não foram encontrados exsicatas desta espécie na coleção Fungi Rickiani do Herbário do Instituto Anchietano de Pesquisas (PACA)....

Importância: Segundo Didukh et al. (2003a), esta espécie possui atividades antigenotóxicas e antimutagênicas.

\section{Cystolepiota seminuda (Lash) Bon Documents Mycolo- giques 6(24): 43. 1976.}

(Fig. 3)

Píleo 1,8-2 cm, branco a levemente rosado, carnoso, convexo-campanulado, umbonado, superfície lisa a pulverulenta, tornando-se rosado-ocre quando tocado. Lamelas livres, concolores ao píleo, mudando de cor ao toque, próximas entre si, lamélulas presentes. Estipe 3,2-4 x 0,2-0,4 cm, central, cilíndrico, branco, fibroso, superfície pulverulenta, base bulbosa. Anel ascendente, branco, membranáceo, posicionado no terço superior. Hábito gregário. Basidiósporos 5,2-6,5 x 2,8-3,7 $\mu \mathrm{m}$, hialinos a méleos, ovais, alguns levemente cilíndricos, hilo excêntrico, sem poro germinativo, pseudoamilóides em reagente de Melzer. Basídios 17,9-20 x 5,2-6,2 $\mu \mathrm{m}$, hialinos, clavados, 2 esterigmas. Pleurocistídios não observados. Queilocistídios claviformes. Camada cortical formada exclusivamente por esferocistos.

Habitat: Crescendo em solo na borda da mata.

Material examinado: Brasil. RJ: Nova Iguaçú, REBIOTinguá, Estrada do Colomi. 22³5'03.4”S-4324'47.4”W, 
alt. $97 \mathrm{~m}$, coletores: M. P. Albuquerque et al. 21-III; 14/X/2004 (RB415685).

Distribuição geográfica: América do Sul: Brasil (Guzmán \& Guzmán-Davalos 1992), Europa: França (Bon, 1976).

Comentários: O material concorda com a descrição de C. seminuda presente em Candusso \& Lanzoni (1990), caracterizando-se pelo píleo pulverulento de cor branca e mutável ao toque. Difere um pouco em relação a esta descrição quanto à forma dos esferocistos estando mais de acordo com a descrição de camada cortical presente em Breitenbach \& Kränzlin (1995) que encontraram células subesféricas a ovóides em amostras desta espécie estudadas da Suíça, muito semelhantes às células da camada cortical observadas neste trabalho. Desse modo, parece que ainda não existe um
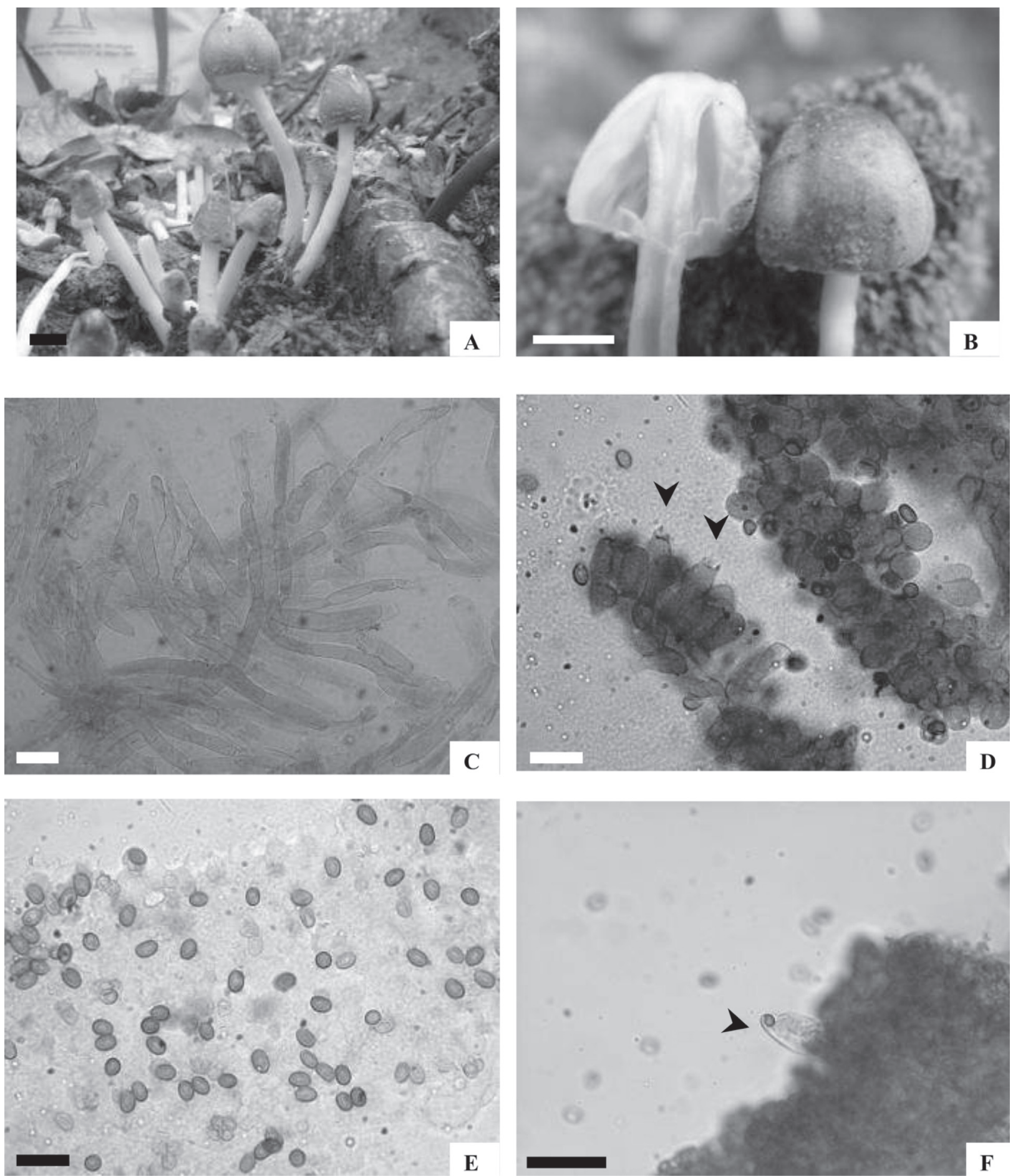

Figura 1. Agaricus dulcidulus Schulzer. A. Hábito. B. Detalhe do píleo e do himenóforo (em corte longitudinal). C. Elementos da camada cortical. D. Basídios (setas). E. Basidiósporos. F. Queilocistídios (setas). (Barra A-B= $1 \mathrm{~cm}, \mathrm{C}=20 \mu \mathrm{m}, \mathrm{D}-\mathrm{F}=10 \mu \mathrm{m}$ ) 


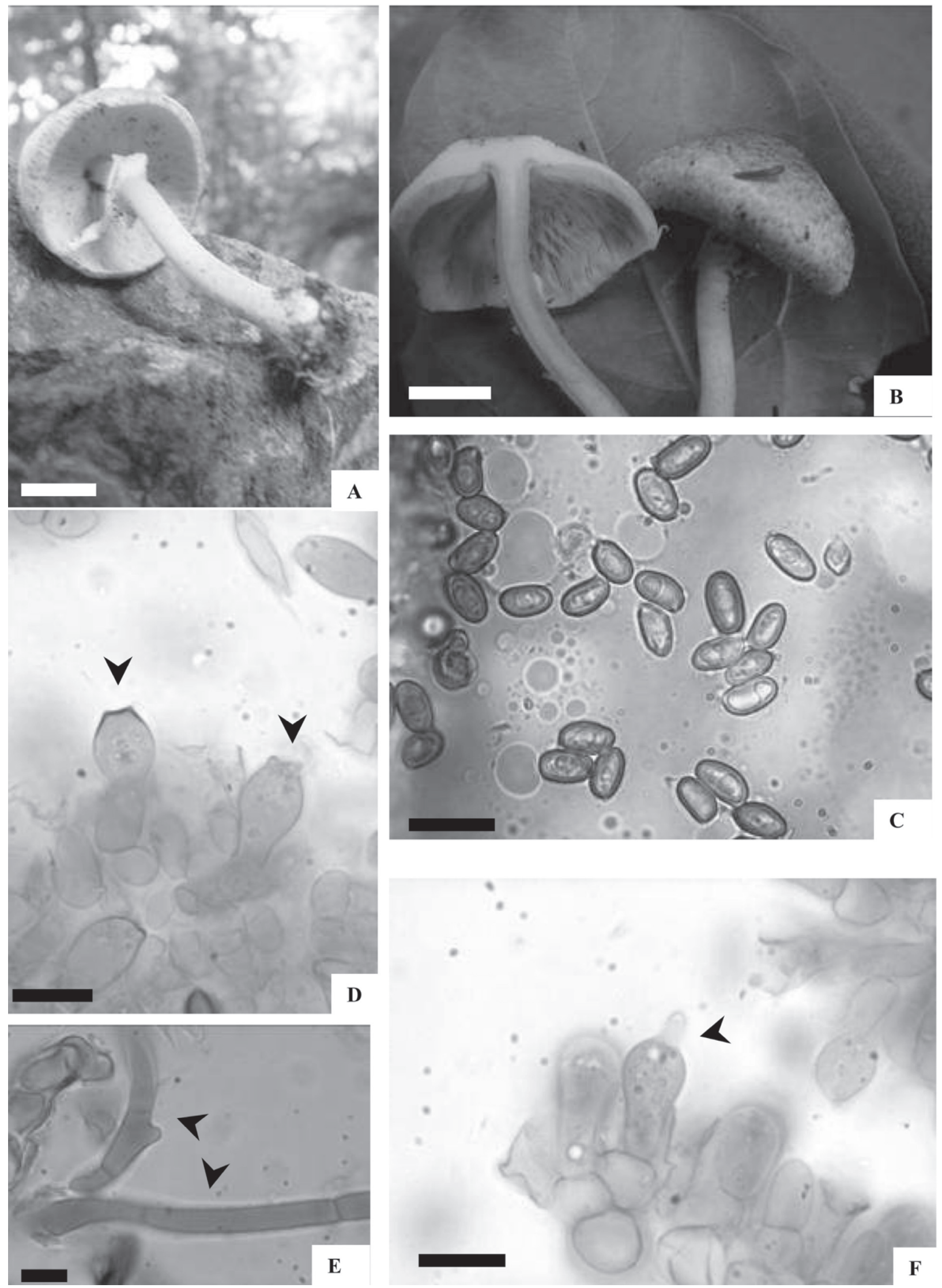

Figura 2. Agaricus subrufescens Peck. A. Hábito. B. Detalhe do píleo e das lamelas. C. Basídios (setas). D. Basidiósporos. E. Hifas da camada cortical (setas). F. Queilocistídios lageniformes (setas). (Barra A-B $=1 \mathrm{~cm}, \mathrm{C}-\mathrm{E}=10 \mu \mathrm{m}$.) 

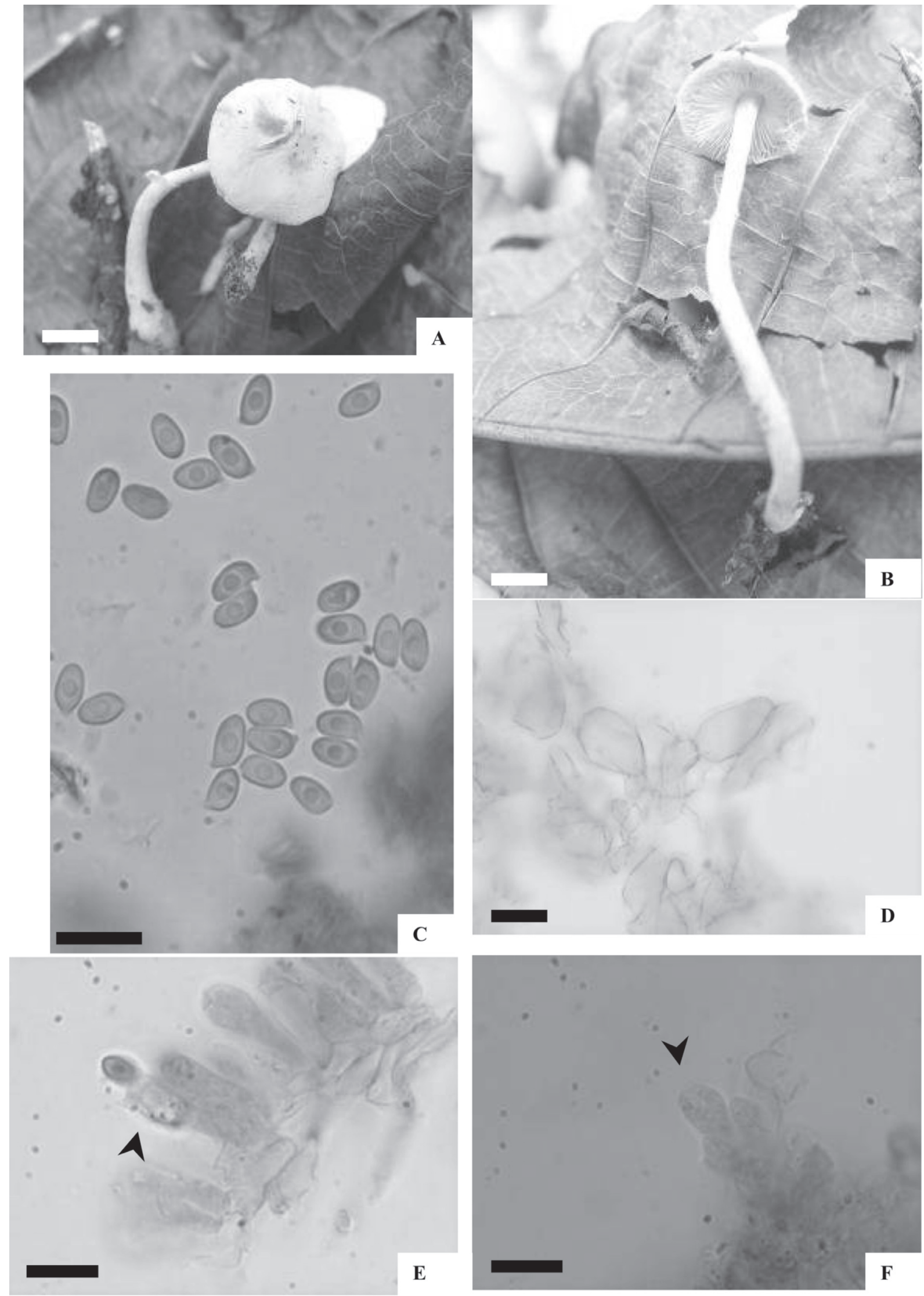

Figura 3. Cystolepiota seminuda (Lash) Bon. A-B. Hábito. C. Basidiósporos. D. Elementos da camada cortical. E. Basídios (seta). F. Queilocistídios (seta) (Barra. $\mathrm{A}-\mathrm{B}=1 \mathrm{~cm}, \mathrm{C}, \mathrm{E}$ e $\mathrm{F}=10 \mu \mathrm{m}, \mathrm{D}=20 \mu \mathrm{m})$. 
conceito amplamente aceito na literatura quanto à forma da camada cortical desta espécie, sendo esta descrita como subesférica, ovóide ou esférica dependendo da literatura.

É possível que o material do presente estudo não tenha hidratado o suficiente dando aspecto subesférico ou que contenha apenas elementos apicais com tendência globosa.

Importância: desconhecida.

\section{Lepiota lilacea Bres., Fung. Trident. 2(8-10): 3. 1892.} (Fig. 4)

Píleo 3,5 cm, branco, carnoso-fibroso, convexo-plano, umbo central com revestimento marrom- púrpura-escuro, liso, formando uma região bem delimitada, superfície recoberta por escamas concêntricas dilacerando-se em direção a margem. Margem do píleo com poucas escamas com um fundo branco, levemente estriada, não mudando de cor ao toque. Lamelas distantes, livres, ventricosas, brancas, imutáveis, lamélulas presentes. Estipe $6,5 \times 0,46 \mathrm{~cm}$, cilíndrico, fistuloso, vináceo, superfície recoberta por poucas fibrilas brancas, de base grossa não bulbosa. Anel persistente, posicionado na região mediana do estipe, fibroso, vináceo, ascendente. Hábito solitário. Basidiósporos 4,3-6x 3,1-4,2 $\mu \mathrm{m}$, hialinos, elípticos, paredes espessas, duplas, podendo ou não ser dextrinóides em reagente de Melzer. Basídios 16 x $5 \mu \mathrm{m}$, difícil visualização, claviformes. Queilocistidios 17-34 x 5,2-12 $\mu \mathrm{m}$, clavados. Camada cortical composta por hifas claviformes 46-90 x 12 $26 \mu \mathrm{m}$, com pigmento marrom, formando uma himeniderme. Fíbulas presentes apenas na camada cortical.

Habitat: Crescendo em serrapilheira, ambiente bastante úmido.

Material examinado: Brasil, Rio de Janeiro: Nova Iguaçú, REBIO-Tinguá, Estrada do Barrelão. 22³3'31.1'”S43⒉'06.2”W; 8/XII/2004 Albuquerque 36-IV, (RB417263), Estrada do Colomi. 2234'11.8"S-43²3'12.8”W; 6/ XII/2004 Albuquerque 7-IV, (RB415687).

Distribuição geográfica: América do Sul: Brasil (Pegler 1997), Venezuela (Dennis 1970). Europa: Itália (Candusso \& Lanzoni 1990).

Comentários: O material foi encontrado no interior da mata, em ambiente de intensa umidade Segundo Candusso \& Lanzoni (1990) no material tipo coletado na Europa, as fíbulas estão presentes em todo o basidioma. Na amostra encontrada na REBIO- Tinguá as fíbulas foram observadas somente na camada cortical. Os basidiósporos da amostra concordam com a descrição do material estudado por Dennis (1961) na Venezuela e Pegler (1997) no Brasil. As descrições de materiais europeus apresentam diferenças na largura dos basidiósporos. Aparentemente os basidiósporos dos espécimes neotropicais são mais elípticos que os encontrados nos espécimes europeus. Lepiota pseudolilacea Huijsman, difere de Lepiota lilacea principalmente em relação ao tamanho dos basidiósporos. L. pseudolilacea apresenta basidiósporos maiores que Lepiota lilacea.

Importância: desconhecida.
5. Lepiota echinella var. rhodorhiza (Romagn. \& Locq. ex P.D. Orton) Hardtke \& Rödel. Mykol. MittBl. 35: 62.1992 (Fig. 5 A-D)

Píleo $1,2 \mathrm{~cm}$, contexto branco, superfície escamosa, marrom, carnoso, plano, umbonado, margem involuta, não mudando de cor ao toque. Lamelas livres, colariadas, brancas. Estipe $3 \times 0,2 \mathrm{~cm}$, cilíndrico, reto, branco, fibroso, não mutável ao toque, base bulbosa $0,4 \mathrm{~cm}$, rizóides presentes, de cor branca. Anel presente, fino, branco, posicionado no terço superior do estipe, ascendente, membranoso, persistente. Hábito solitário. Basidiósporos 5,2-7,3 x 2,6-4 $\mu \mathrm{m}$, hialinos, ovais, obtusos, parede dupla, dextrinóides em reagente de Melzer. Basídios 10-20 x 5-5,5 $\mu \mathrm{m}$, claviformes a subcilíndricos, hialinos, com 4 esterigmas, esterigmas medindo 3 $\mu \mathrm{m}$. Pleurocistídios 11-21 x 5,3-8 $\mu \mathrm{m}$, claviformes, hialinos. Queilocistídios 16-21 x 6,5-8 $\mu \mathrm{m}$. Camada cortical formada por elementos de dois tipos, uma camada himeniforme com elementos claviformes, subglobosos a esferopedunculados, hialinos, medindo 20-53 x 7,1-14 $\mu \mathrm{m}$ situados na base de hifas eretas, longas, com pigmento marrom ou hialinas, medindo 84,6-168,9 x 6,5-12 $\mu \mathrm{m}$. Fíbulas presentes na camada cortical.

Habitat: Crescendo em solo em interior de mata.

Material examinado: Brasil. Rio de Janeiro: Nova Iguaçú, REBIO-Tinguá, Estrada do Colomi. 22³5'03.4”'S43⒉47.4”W, alt. 97m, 14/X/2004 Albuquerque 41-III, (RB417269). Distribuição geográfica: América do Sul: Brasil (neste trabalho). Europa: França, Itália, Liechtenstein (Candusso \& Lanzoni 1990).

Comentários: Os elementos da camada cortical, forma e tamanho dos basidiósporos e a presença de rizóides na base do estipe, possibilitam determinar a amostra como pertencente à seção Ovisporae do gênero Lepiota. Segundo Candusso \& Lanzoni (1990), L. echinella var rhodorhiza (como L. rhodorhiza) apresenta anel débil ou ausente. No presente estudo a amostra possui traços bem evidentes do anel, percebendo-se sua orientação ascendente, os demais caracteres se encaixam perfeitamente na descrição apresentada pelos autores citados acima. Migliozzi \& Coccia (1990), consideram esta uma espécie muito comum no mediterrâneo.

Importância: desconhecida.

\section{Lepiota forquignonii var. forquignonii Quél., Comptes} rendu Assoc. Franc. Avanc.

Sci. 13: 277. 1885.

(Fig. 5 E-G)

Píleo $2 \mathrm{~cm}$, branco, carnoso, aplanado, sub-umbonado, superfície recoberta por escamas de coloração laranja, disco central mais escuro, margem esbranquiçada. Lamelas livres, brancas, lamélulas presentes, subdistantes, não mudando de cor ao toque, borda da lamela fértil. Estipe $4 \times 0,3 \mathrm{~cm}$, branco, cilíndrico, levemente curvado, membranoso, não mudando de cor ao toque, recoberto no terço basal por 

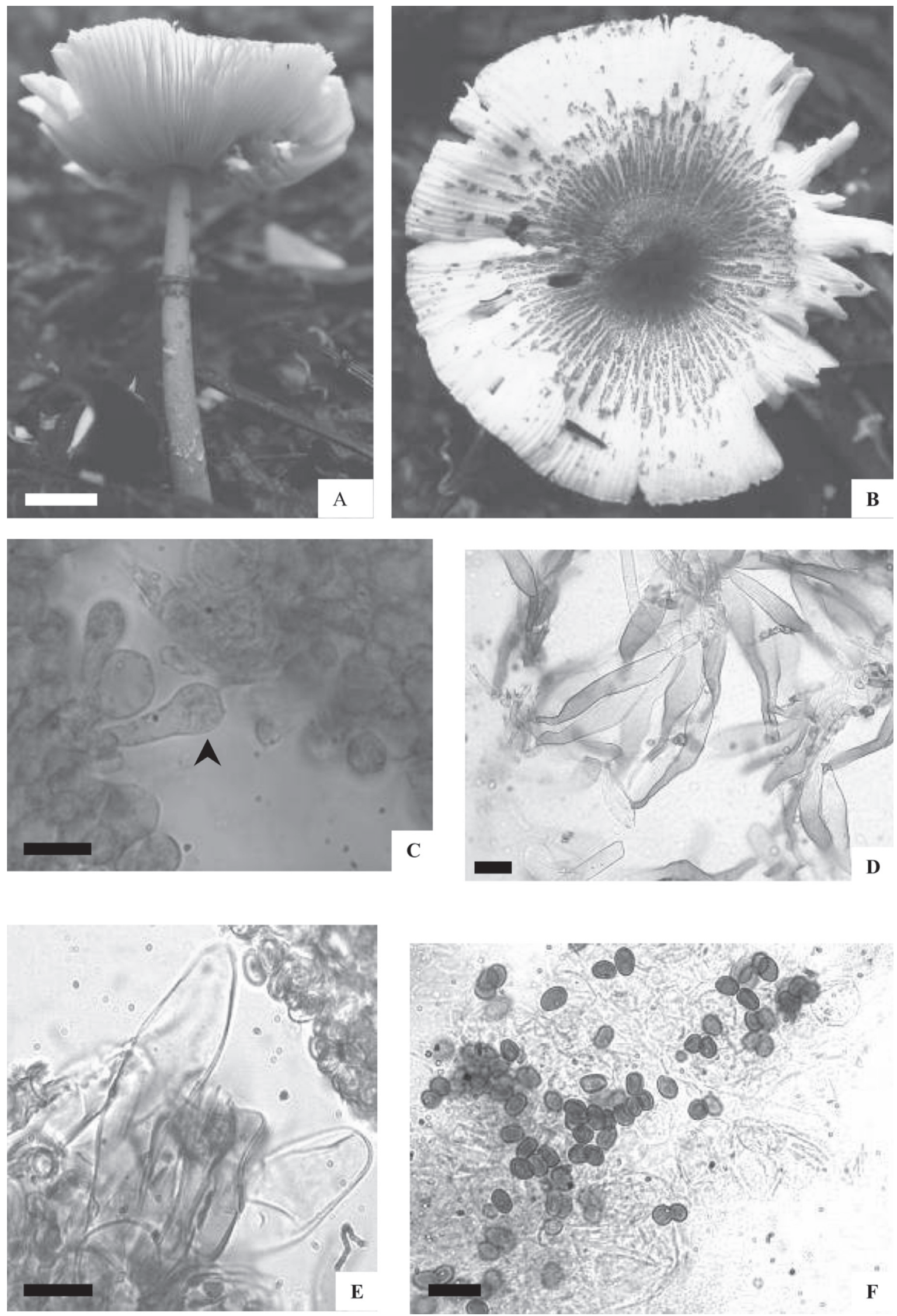

Figura 4. Lepiota lilacea Bres. A. Hábito. B. Detalhe da superfície do píleo. C. Basídio (seta). D. Elementos da camada cortical. E. Queilocistídios claviformes. F. Basidiósporos em Melzer. (Barra A=1 cm, C, E e F=10 $\mu \mathrm{m}, \mathrm{D}=20 \mu \mathrm{m}$ ). 


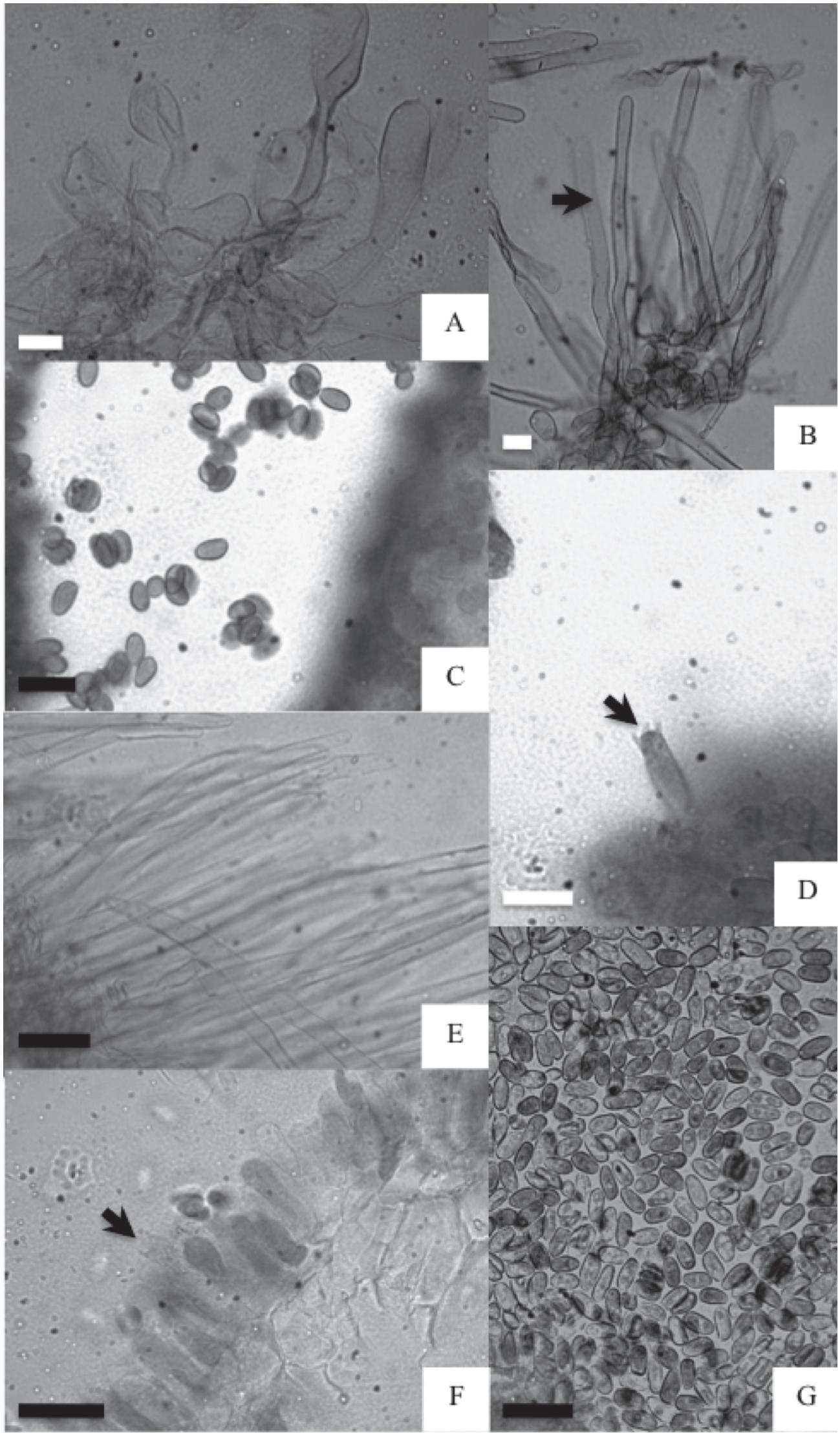

Figura 5. A-D Lepiota echinella var rhodorhiza (Romagn. \& Locq. ex P.D.Orton) Hardtke \& Rödel. A. Elementos himeniformes da camada cortical do píleo. B. Elementos tricodermais da camada cortical do píleo (seta). C. Basidiósporos. D. Basídio (seta). E-F. Lepiota forquignonii var forquignonii Quél. E. Camada cortical

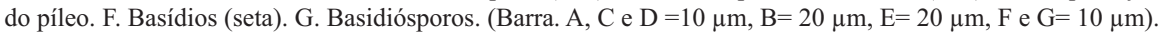


escamas concolores a superfície do píleo, base não bulbosa. Sem vestígios de anel. Hábito solitário. Basidiósporos 6,0-7,7 x 2,8-3,9 $\mu \mathrm{m}$, hialinos, elípticos, parede dupla, sem poro germinativo, com apêndice hilar excêntrico bastante pronunciado, uninucleado ou binucleado, dextrinóide em reagente de Melzer. Basídios 10-18 x 5,1-7,2 $\mu$ m claviformes, hialinos, com 4 esterigmas. Pleurocistídios ausentes. Queilocistídios 16-27 x 6,1-9,1 $\mu \mathrm{m}$, hialinos, cilíndricos, ventricosos. Camada cortical 115-260 x 7,7-15 $\mu \mathrm{m}$, composta por hifas eretas, finas, longas, hialinas a marrom, algumas bifurcadas, formando uma paliçada, às vezes lembrando uma plectocútis. Fíbulas presentes na camada cortical.

Hábitat: Crescendo sobre madeira na borda da mata.

Material examinado: Brasil. Rio de Janeiro: Nova Iguaçú, REBIO-Tinguá, Estrada do Ouro. 22³6'31.7'S$43^{\circ} 28^{\prime} 08.0^{\prime \prime} \mathrm{W}$, alt. $774 \mathrm{~m}, 5 / \mathrm{XII} / 2003$ Albuquerque et al. 49-I; (RB417274).

Distribuição geográfica: África: Não é citado o país (Bon, 1981). América do Norte: EUA. (Guzmán \& GuzmánDavalos, 1992). América do Sul: Brasil (Rick, 1937) Europa: França, Itália (Candusso \& Lanzoni, 1990).

Comentários: A amostra apresenta camada cortical e basidiósporos característicos da seção Ovisporae, subseção Felininae (Bon 1981). Comparada com as espécies européias, principalmente as estudadas por Candusso \& Lanzoni (1990), a amostra se enquadra na descrição de Lepiota forquiginonii var. forquiginonii excetuando-se o fato da amostra obtida neste trabalho ocorrer sobre madeira. Segundo Bon (1981) Lepiota forquiginonii var. forquiginonii assemelha-se a L. rhodorhiza var speciosa, mas pode ser diferenciada pelo tamanho e coloração do píleo e estipe e microscópicamente por apresentar basidiósporos maiores que os de Lepiota forquiginonii var. forquiginonii.

Importância: desconhecida.

\section{Lepiota subincarnata Lange, Fl. Agaric. Danic. 5 (Appx.):} V. 1940.

(Fig. 6)

Píleo 1,5 cm, branco, carnoso, plano-convexo, centro do píleo escuro subumbonado, com escamas marrons, furfuráceas, diminuindo em direção a margem. Lamelas livres de coloração branca. Estipe 1,6 x 0,1 cm cilíndrico, base levemente curvada, engrossada $0,3 \mathrm{~cm}$, inicialmente branco, apresentando a superfície escamosa marrom em direção a base. Anel não evidente. Hábito solitário. Basidiósporos 5,6-8,2 x 3,2-4,0 $\mu \mathrm{m}$, hialinos, elípticos a ovais, dextrinóides em reagente de Melzer. Basídios 10,9-16,3 x 5,5-7,5 $\mu \mathrm{m}$, hialinos, clavados, com 4 esterigmas. Pleurocistídios não observados. Queilocistídios não observados. Camada cortical formada por uma himenotricoderme, composta por hifas finas, alongadas e subcilíndricas 50-198 x 8-50 $\mu \mathrm{m}$, com algumas hifas bifurcadas. Fíbulas presentes na camada cortical. Habitat: Crescendo no solo no interior da mata.

Material examinado: Brasil. Rio de Janeiro: Nova
Iguaçú, REBIO-Tinguá, Estrada do Colomi. 22³5'03.4”S4324'47.4'W, alt 80m 15/X/2004 Albuquerque, Silva 40III, (RB417267).

Distribuição geográfica: América do Sul: Argentina (Wrigth \& Albertó, 2002). Brasil (neste trabalho). Europa: França, Itália (Candusso \& Lanzoni, 1990).

Comentário: Os elementos alongados da camada cortical, forma dos basidiósporos e características macroscópicas da amostra coletada na REBIO-Tinguá permitem determina-lá como L. subincarnata. Segundo Candusso \& Lanzoni (1990), L. subincarnata pertence a seção Ovisporae, apresentando caracteres comuns a $L$. josserandi Bon \& Boiffard (=L.subincarnata) e L. helveola . Bon \& Boiffard (1974), apresentam as diferenças destas espécies sendo relevante para separação os elementos da camada cortical, (comprimento em torno de $200 \mu \mathrm{m}$ e hifas rígidas em $L$. subincarnata, e em torno de $130 \mu \mathrm{m}$ e hifas flácidas em L. helveola), outros caracteres importantes para diferenciação destas espécies são: relação existente entre o comprimento do estipe e diâmetro do píleo e o tipo de ornamentação do estipe, esquamuloso ou flocoso em $L$. subincarnata e liso em $L$ helveola. Os caracteres macroscópicos e microscópicos se encaixam com as descrições de $L$. subincarnata apresentadas em Candusso \& Lanzoni (1990) e em Wrigth \& Albertó (2002), nesse último como L. josserandii.

Importância: Segundo Wrigth e Albertó (2002) em caso de ingestão esta espécie pode causar intoxicação grave.

\section{Lepiota tepeitensis Murril Mycologia 3: 82. 1911.}

\section{(Fig. 7)}

Píleo 6,2 cm, esbranquiçado, convexo-aplanado, carnoso, umbo presente, evidente, marrom escuro, superfície do píleo recoberta por escamas marrom avermelhadas, dilacerando-se em direção a margem, estrias presentes apenas na margem. Lamelas livres, remotas, creme pálidas. Estipe 9,4 x 0,3 cm, bege avermelhado, cilíndrico, reto, fibroso, fistuloso, com base bulbosa $0,8 \mathrm{~cm}$, com rizóides. Anel membranoso, simples, móvel. Hábito solitário. Basidiósporos 4,3-6,7 x 2,9$4,4 \mu \mathrm{m}$, hialinos, elipsóides a ovais, sem poro germinativo, reação com Melzer não observada, hilo excêntrico. Basídios não observados. Queilocistídios 38-67 x 11-16 $\mu \mathrm{m}$, claviformes a ventricosos com longos apêndices. Pleurocistidios $46 \times 36,5 \mu \mathrm{m}$, esferopedunculados, com pigmento marrom. Camada cortical 35-134 x 10-21 $\mu$, formada por elementos eretos de diferentes formas semelhantes à queilocistídios, podendo ser clavados, utriformes, ventricosos, formando uma tricoderme. Fíbulas ausentes.

Habitat: Crescendo em solo na borda da mata

Material examinado: Brasil. Rio de Janeiro: Nova Iguaçú, REBIO-Tinguá, Estrada do Colomi. 22034'01.7'S$43^{\circ} 22^{\prime} 47.9^{\prime} \mathrm{W}$, alt. $51 \mathrm{~m}, 30 / \mathrm{VI} / 2004$ Albuquerque 4-II; (RB417242), Estrada do Colomi. 22036'31.7'S$43^{\circ} 28^{\prime} 08.0$ "W, alt. 772m, 6/XII/2004 Albuquerque 75-I; (RB418368). 
Distribuição geográfica: América central: México (Murril, 1911). América do Sul: Brasil (Capelari, 1989). Caribe: Martinica (Pegler, 1983).

Comentários: Com exceção dos queilocistídios que na amostra são maiores que os descritos por Pegler (1983), medindo 23-34 x 8-12 $\mu \mathrm{m}$ no material estudado por este autor, e 38-67 x 11-16 $\mu \mathrm{m}$ na amostra da REBIO-Tinguá, as demais características microscópicas e macroscópicas concordam fortemente com a descrição de Pegler. Capelari (1989) cita pela primeira vez esta espécie para o Brasil, sob $\mathrm{n}^{\circ}$ de exsicata SP193829, coletado na Ilha do Cardoso, São Paulo. Pegler (1997) revisando este material o identificou como Lepiota serena (Fr.) Sacc. Bon e Boiffard (1974) transfere Lepiota serena para o gênero Leucoagaricus. Heinemann (1978) faz uma nova combinação com L. serena criando o gênero Sericeomyces. A amostra descrita no presente trabalho difere de L. serena devido à dimensão e coloração do píleo, tamanho dos basidiósporos (pouco menores em L. tepeitensis), mas difere principalmente pela camada cortical que em L. tepeitensis é constituída por hifas eretas e em L. serena constítue-se de hifas prostradas com poucos elementos eretos. Capelari (1989) ao descrever a camada cortical de seu material, um espécime de $L$. tepeitensis, observa elementos eretos contendo pigmento marrom, conforme descrição de L. tepeitensis disponível em Murril (1911) e Pegler (1983).

Importância: desconhecida.

\section{Referências Bibliográficas}

Bon, M. \& Boiffard, J. 1974. Lepiotes de vendèe et de la corte Atlantique Française. I. Bulletin du Societé Mycologique de France 90(4): 287-306.

Bon, M. 1981. Cle monographique des "lepiotes" d' europe (= Agaricaceae, tribus Lepiotae et Leucocoprineae). Documents Mycologiques. 11(43): 1-77.

Candusso, M. \& Lanzoni, G. 1990. Lepiota s.l. Saronno, Itália. Libréria Editrici Gionana Bielle.

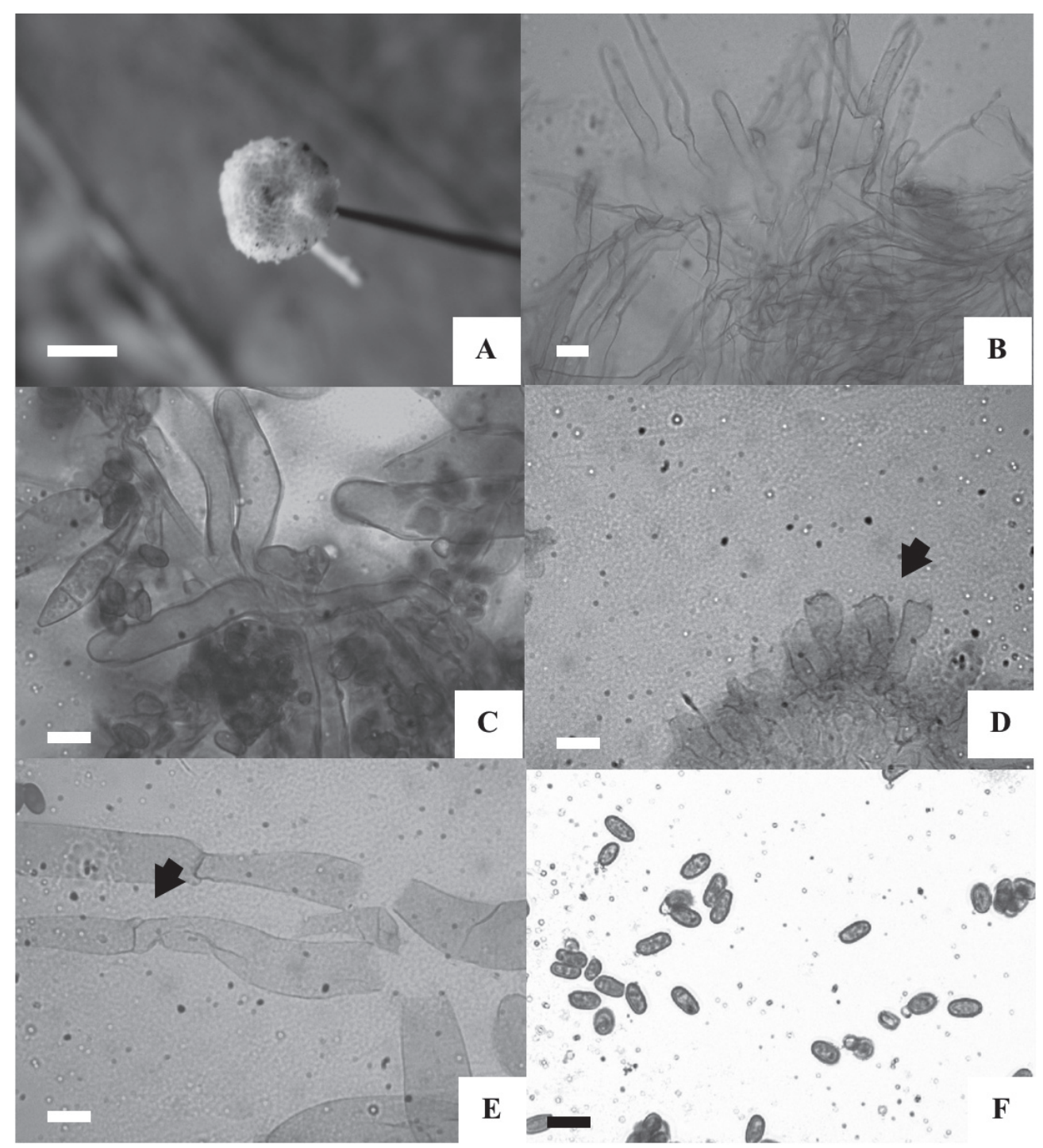

Figura 6. Lepiota subincarnata Lange. A. Hábito. B. Camada cortical do píleo. C. Detalhe dos elementos da camada cortical. D. Basídios (seta). E. Fíbulas nos septos dos elementos da camada cortical (seta). F. Basidiósporos (Barra. A =1 cm B= $20 \mu \mathrm{m}, \mathrm{C}-\mathrm{F}=10 \mu \mathrm{m}$ ). 


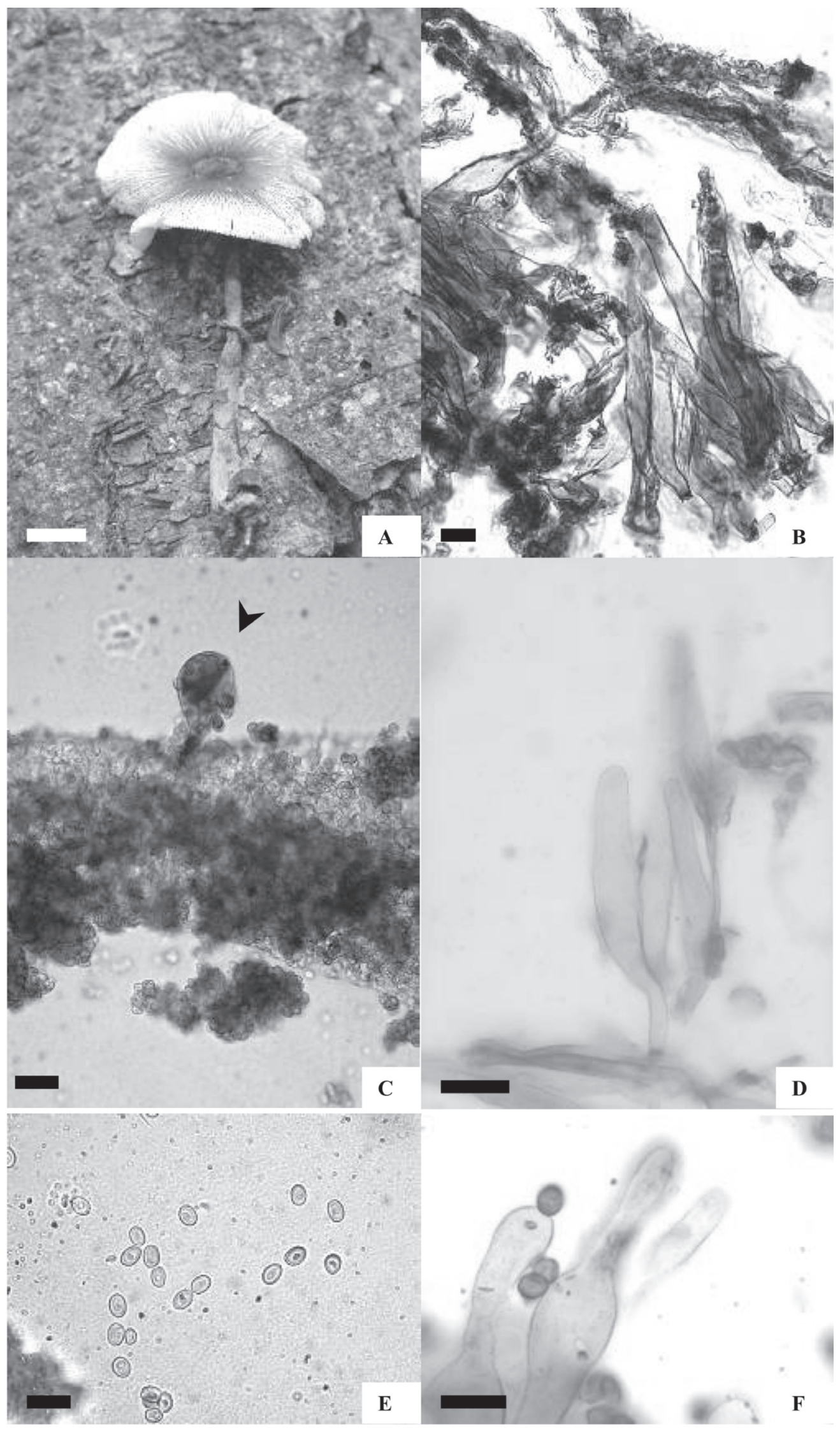

Figura 7. Lepiota teipeitensis Murril. A. Hábito. B. Camada cortical do píleo. C. Pleurocistídios (seta). D. Elementos não pigmentados da camada cortical do píleo. E. Basidiósporos. F. Queilocistídios. (Barra A=1 cm, B e D=20 $\mu \mathrm{m}, \mathrm{C}, \mathrm{E}$ e F=10 $\mu \mathrm{m}$ ). 
Capelari, M. 1989. Fungos Agaricales da Ilha do Cardoso (exceto Tricholomataceae). Dissertação de mestrado. Universidade de São Paulo, São Paulo.

Cappelli, A. 1984. Fungi europaei. Agaricus I ex Fr. ss Karsten (Psaliota Fr.). Soronno, Libreria Edritrice Giovanna Bielle.

Dennis, R. W. G.1961. Fungi venezuelani:IV. Agaricales. Kew Bulletin 15: $67-156$.

Didukh, M. Ya., Wasser, S. P. \& Nevo, E. 2003. Medicinal value of species of the family Agaricaceae Cohn (Higher Basidiomycetes): Current stage of knowleadgeand future perspectives. International Journal of Medicinal Mushrooms 5: 133-152.

Eira, A. F. Fungos Comestíveis. Pp379-447. In: Elisa Esposito, E. e Azevedo J. L. 2004. Fungos: uma introdução à biologia, bioquímica $e$ biotecnologia. Caxias do Sul. Educs.

Golfari, L. \& Moosmayer, H. 1980. Manual de reflorestamento do estado do Rio de Janeiro. Rio de Janeiro, Secretaria de Planejamento e Coordenação Geral.

Guedes-Bruni, R. R., Pessoa, S. V.A. \& Kurtz, B. 1997. Florística e estrutura do componente arbustivo-arbóreo de um trecho preservado de floresta montana na Reserva Ecológica de Macaé de Cima. Pp. 127-145.In: Lima, H. C. de \& Guedes-Bruni, R. R. (eds.). 1997. Serra de Macaé de Cima: Diversidade florística e conservação em Mata Atlântica. Jardim Botânico do Rio de Janeiro, Rio de Janeiro.

Guzmán, G.\& Guzmán-Dávalos, L. 1992. A checklist of the Lepiotaceus Fungi. Champaing, Koeltz Scientific Books.

Heinemann, P. 1978. Seriomyces, genre nouveau de Leucocoprineae (Agaricaceae). Bulletin Jardin Botaniche Nat. Belg. 48: 399-407.

Heinemann, P. 1993. Agarici Austroamericani VIII. Agariceae des regions intertropicals d'Amerique du Sud. Bull. Jard. Bot. Nat. Belg. 62: 355-384.

Heinemann, P. Agarici Austroamaricani IV. Quatre Agaricus du Venezula. Bull. Jard. Bot. Etad. Brux. 32: 155-161.

IPJBRJ, 2002. Projeto Tinguá. Paisagem e flora da reserva biológica do Tinguá: subsídios ao monitoramento da vegetação. Relatório técnico-cientifico. Rio de Janeiro, Instituto de Pesquisas Jardim Botânico do Rio de Janeiro/Universidade Federal Rural do Rio de Janeiro.

Kerrigan, R.W. 2005. Agaricus subrufescens, a cultivated edible and medicinal mushroom and its synonyms. Mycologia 97, 12-24.22.

Kerrigan, R. E. \& Ross, I. K. 1988. Extracelular laccases: biochemical makers for Agarius systematics. Mycologia, 80: 689-695.

Kirk, P. M., Cannon, P. F., David, J. C. \& Stalpers, J. A. 2001. Ainsworth \& Bisby's Dictionary of the Fungi, 9th. Wallingford, CAB International.

Koeppen, W. 1948. Climatologia. México, Fundo de Cultura Econômica.

Migliozzi, V. \& Coccia, M. 1990. Lepiota hymenoderma, Lepiota rhodorhiza, Lepiota speciosa, Leucoagaricus tener forma. Fungi del Lazio. II. 6-10. Micologia Italiana 19(1): 11-24.
Murril, W. A. 1911. The Agaricaceae of tropical North America II. Mycologia 3: 79-91.

Nauta, M. M. 2001. Genus Agaricus. Pp. 64-151, In: Noordeloos, M.E.; Kuyper, TH.W.; Vellinga, E.C. Flora Agaricina Neerlandica, 5. Tokyo A.A. Balkema Publishers, Lisse, Abingdon, Exton (PA).

Pegler, 1983. Agaric flora of Lesser Antilles. Kew Bull. Add. Ser. IX

Pegler, 1997. The Agarics of São Paulo. An account ot the agaricoid fungi (Holobasidiomycetes) of São Paulo State, Brazil. Kew, Royal Botanic Gardens.

Pereira \& Putzke, J. 1990. Famílias e gêneros de fungos Agaricales (cogumelos) no Rio Grande do Sul. Santa Cruz do Sul, ed. Fisc.

Radam-Brasil. 1983. Folhas sf 23/24 Rio de Janeiro/Vitória: geologia, geomorfologia, pedologia, vegetação e uso da terra. Rio de Janeiro, RADAM - Brasil.

Ribeiro, J. E. L. da S., Hopkings, M. L. G., Vicentini, A., Sothers, C. A., Costa, M. A.. da S., Brito, J. M. de, Souza, M. A. D. de, Martins, G.

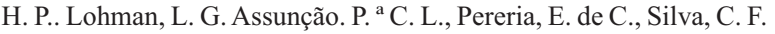
da, Mesquita, M. R., Procópio, L. C. 1999. Flora da Reserva Ducke. Guia de identificação das plantas vasculares de uma floresta de terra-firme na Amazônia Central. Manaus, INPA/DFID.

Rick, J.. 1930. Contributio ad monographiam agaricacearum brasiliensium IV. Broteria 24: 97-118.

Rick, J.. 1939. Agarici Riograndensis IV. Lilloa 4: 75-104

Saracura, V. F.1996. Reserva biológica do Tinguá. Plano de ação emergencial. Brasília, Ministério do Meio Ambiente, dos Recursos Hídricos e da Amazônia Legal.

Silva, M da \& Esposito, E. O papel dos fungos na recuperação ambiental. Pp. 337-378. In: Esposito, E. \& Azevedo J. L. 2004. Fungos: uma introdução à biologia, bioquímica e biotecnologia. Caxias do Sul, Educs.

Singer, 1986. The Agaricales in modern taxonomy. $4^{\mathrm{a}}$ ed. Koenigstein. Koeltz Scientific Books.

Veloso, H. P.; Rangel Filho, A. L. R.; Lima, J. C. A. 1991. Classificação da vegetação brasileira, adaptada a um sistema universal. Rio de Janeiro, IBGE, Departamento de Recursos Naturais e Estudos Ambientais.

Wasser, S. P. 2002. Family Agaricaceae (Fr.) Conh (Basidiomycetes) of Israel Mycobiota. I. Tribe Agariceae Pat. A. R.G. Ruggell, Verlag Kommandit Gesellschaft.

Wasser, S. P., Didukh, M. Ya, de Amazonas, M. A. L., Nevo, E., Stamets, P. \& da Eira, A. F. 2002. Is widely cultivated culinary-medicinal Royal Sun Agaricus (the Himematsutake mushroom) indeed Agaricus blazei Murril? International Journal of Medical Mushroom. 4: 267-290.

Wright, J. \& Albertó, E. 2002. Hongos. Guia de la region pampeana I. Hongos com laminillas. Buenos Aires. L.O.L.A. 